\title{
Anforderungen an die Fernerkundung und die Abfluss- modellierung aus Sicht der wasserwirtschaftlichen Planung
}

Zusammenfassung: Airborne Laserscanning Daten stellen eine wesentliche Verbesserung für die Erfassung und Modellierung von Hochwasserereignissen dar. Eine Herausforderung ist dabei die korrekte Filterung der Punktwolke in Oberflächen- und Geländepunkte, sowie die repräsentative Ausdünnung und Verknüpfung mit der terrestrischen Vermessung als Basis für das hydraulische Berechnungsmodell. Die Überprüfung der Laserscanning Daten auf die geforderte Lage- und Höhengenauigkeit stellt dabei eine Voraussetzung für die effiziente Anwendung dar.

Intended purposes of remote sensing and flow modelling in terms of water resources planning

Summary: Airborne laser scanning data afford a considerable enhancement for recording and modelling flood events. The great challenge presented by this method is the correct filtering of the point cloud into surface and terrain points and the representative thinning and linkage with terrestrial surveys as a basis for the hydraulic computation model. Efficient application of this method, however, calls for a check to be made of the laser scanning data for the required accuracy of location and levels.

\section{Ausweisung von \\ Hochwasserabflussgebieten}

In der Steiermark werden seit Anfang der 90er Jahre für Gewässer im Zuständigkeitsbereich der Bundeswasserbauverwaltung Hochwasserabflussgebiete für ein 30-jährliches und ein 100-jährliches Hochwasserereignis ausgewiesen. Derzeit liegen für rund $2.000 \mathrm{~km}$ Gewässer Hochwasseranschlaglinien vor. Im Zuge eines Sonderprogramms zur Ausweisung und Aktualisierung von Hochwasserabflussgebieten wurden im Jahr 2008 2DHochwasserabflussuntersuchungen für rund $450 \mathrm{~km}$ Fließgewässer fertig gestellt. Die Ergebnisse der Abflussuntersuchungen sowie erste technische Maßnahmenkonzepte wurden den betroffenen $\mathrm{Ge}$ meinden präsentiert und dienen als Grundlage für alle weiteren Behördenverfahren sowie für die zukünftige Raumplanung in jeder Gemeinde. Weitere 2DHochwasserabflussuntersuchungen für rund $400 \mathrm{~km}$ Fließgewässer sind derzeit in Bearbeitung und werden im Jahr 2010 fertig gestellt (Abb. 1)

Bei 2D-Modellierungen werden in der Regel Airborne Laserscanning (ALS) Daten verwendet, die durch Befliegungen des Projektgebietes ermittelt werden. Durch das daraus resultierende genaue Geländemodell sind parzellenscharfe Ausweisungen der Wassertiefen sowie der Fließgeschwindigkeiten möglich. Jedes Modell kann jedoch nur ein vereinfachtes, abstraktes Abbild der Realität darstellen. Das heißt, dass trotz der sehr genauen Analyse und Darstellung der Abflusssituation niemals alle Einflussfaktoren berücksichtigt werden können und somit immer ein Risiko für zusätzliche Abflussbereiche - z. B. bei Verklausung von Brücken und Durchlässen, Geschiebeeintrag, Dammbruch, etc. - verbleibt. Deshalb ist es wesentlich, die Bevölkerung in möglichen Gefährdungsbereichen mittels aktueller Informationen über Naturgefahren zu sensibilisieren, um eine entsprechende Risikowahrnehmung und Risikoakzeptanz zu erreichen.

\section{Anforderungen an die ALS Daten hinsichtlich der Beschaffung}

Im Zuge der Erstellung von 2D Hochwasserabflussuntersuchungen wurden im Jahr 2006 Airborne-Laserscanning (ALS) Befliegungen gemeinsam mit anderen Dienststellen (Straßenbau, Wildbach-und Lawinenverbauung, u. a.m.) für Teilbereiche der Steiermark ausgeschrieben und durchgeführt. Eine flächendeckende ALS Befliegung für die Steiermark ist bis 2010/ 2011 geplant.
Diesbezüglich wird angemerkt, dass sich die Möglichkeiten der Airborne Laserscanning Daten rasant entwickeln und laufend verbessern. Bei den ersten ALS-Befliegungen wurden mittlere Punktdichten von 1 Punkt je $4 \mathrm{~m}^{2}$ (mittlerer Punktabstand ca. $2 \mathrm{~m}$ ) erhoben. Bei den obigen Befliegungen wurde bereits 1 Punkt je $\mathrm{m}^{2}$ gefordert und bei aktuellen Projekten werden in den Talräumen meistens 4 Punkte je $\mathrm{m}^{2}$ erfasst. Die erreichten Genauigkeiten wurden in den letzten Jahren ebenfalls verbessert - so ist der mittlere Höhenfehler von $+/-20 \mathrm{~cm}$ auf $+/-15 \mathrm{~cm}$ verbessert worden, wobei mit höherem Aufwand bei der Transformation +/- $10 \mathrm{~cm}$ erreichbar sind.

Eine wesentliche Verbesserung in der Qualität der Datenfilterung kann heute durch den Einsatz von full-waveform Laserscannern erreicht werden (Mandlburger et al. 2009). Bei diesen modernen Instrumenten werden nicht nur bis zu vier diskrete Punkte je Messung (First- and last Echo und zwei weitere Reflexionen) registriert, sondern das gesamte reflektierte $\mathrm{Si}$ gnal wird aufgezeichnet. Durch die zusätzliche Information der Pulsbreite sind wesentliche Verbesserungen bei der Datenfilterung möglich.

Die Vergabe der ALS-Befliegungen Steiermark 2006 für potentielle Hochwasserabflussgebiete erfolgte nach dem Bestbieterprinzip. Zuschlagskriterien waren der Gesamtpreis, die Messmethodik und das Lieferdatum. Die Kosten lagen in Abhängigkeit des zu befliegenden Bereiches zwischen €300,- und €400,- pro $\mathrm{km}^{2}$. Die Ausschreibung umfasste folgende Leistungen:

1. Durchführung einer ALS Befliegung

2. Berechnung einer 3D-Punktwolke

3. Klassifizierung in Oberflächen- und Geländepunkte

4. Modellierung eines Digitalen Geländemodells $1 \mathrm{~m}$ x $1 \mathrm{~m}$

5. Modellierung eines Digitalen Oberflächenmodells $1 \mathrm{~m}$ x $1 \mathrm{~m}$

Anforderungen:

- Mittlere Punktdichte von 1 Punkt pro $\mathrm{m}^{2}$ und ein maximaler Punktabstand 


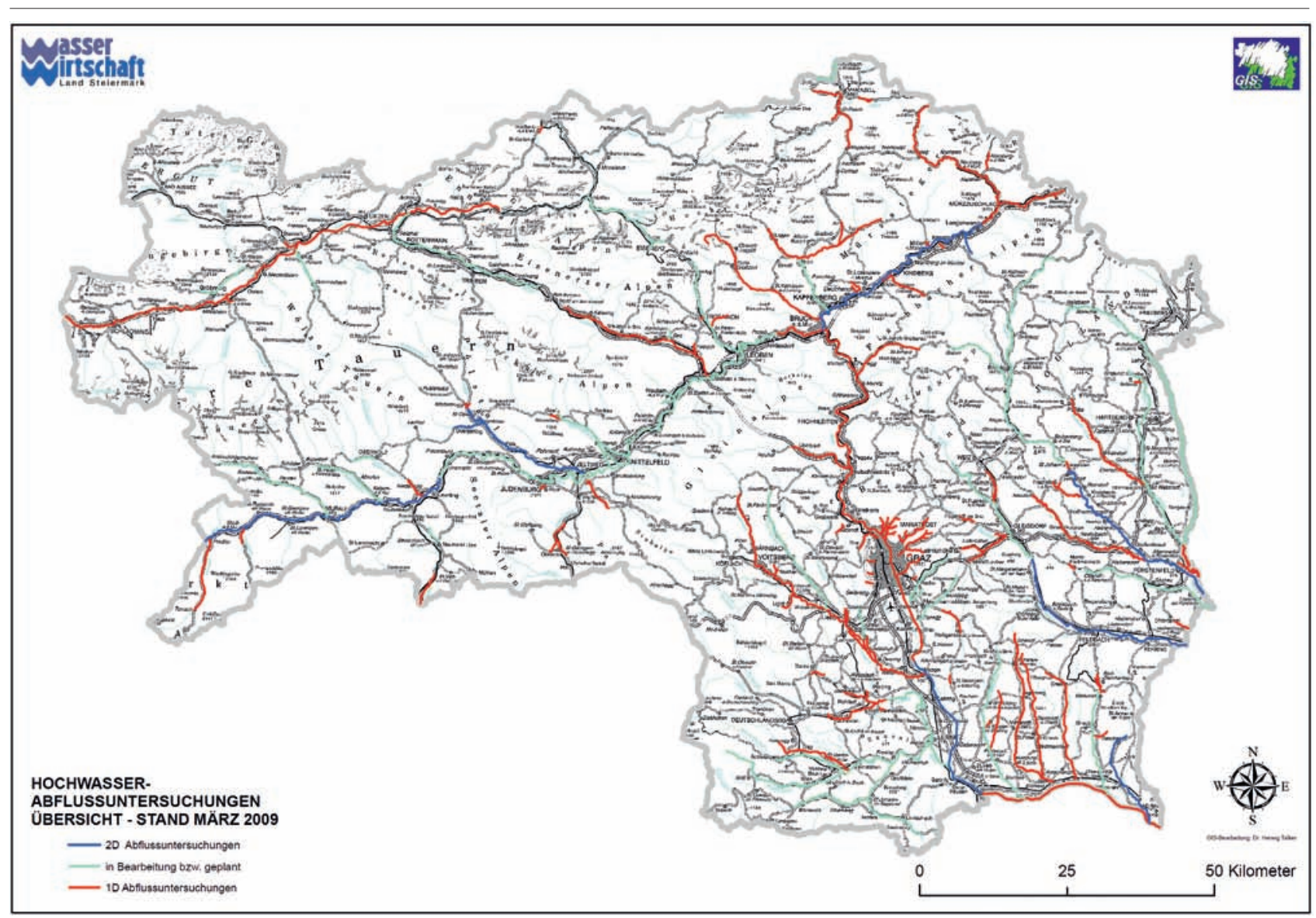

Abb. 1: Übersichtskarte der Abflussuntersuchungen in der Steiermark.

von 1,5 $\mathrm{m}$ in Längs- und Querrichtung der gemessenen Punkte

- Lagegenauigkeit besser $+/-50 \mathrm{~cm}$

- Höhengenauigkeit besser $+/-15 \mathrm{~cm}$

- Befliegungszeitpunkt wenn Projektgebiete trocken und schneefrei sind, sowie die Vegetation ohne Belaubung ist

- GPS-Referenzdaten während der Befliegung

- Vermessung von Kontrollflächen

\section{Lieferumfang:}

1. Metadaten der Befliegung: Lage der Flugstreifen und Grenzen des Aufnahmebereiches

2. Ungefilterte 3D-Punktwolke: Georeferenzierte Punktwolke, bei der alle bekannten Gerätekorrekturen angebracht und die absolute Höhen- und Lageanpassung vorgenommen wurde

3. Geländemodell (nur Bodenpunkte): transformierte und gefilterte Punktwolke

4. Transformationsparameter, Topographien der GPS-Bodenpunkte

5. Digitales Geländemodell: gefilterter und interpolierter Punktraster $1 \mathrm{~m} \times 1 \mathrm{~m}$
6. Digitales Oberflächenmodell: gefilterter und interpolierter Punktraster $1 \mathrm{~m} \times 1 \mathrm{~m}$

Das Digitale Geländemodell (DGM, engl. DTM - Digital Terrain Model) ist ein digitales, numerisches Modell, welches die Geländehöhe der Erdoberfläche beschreibt. Das Digitale Oberflächenmodell (DOM, engl. DSM - Digital Surface Model) hingegen beschreibt die oberste Fläche aus der Vogelperspektive. Im offenen Gelände ist das DOM gleich dem DGM, bei Häusern läuft es aber über die Dachfläche, und im bewaldeten Gebieten wird die Oberfläche durch die in einander greifenden Baumkronen gebildet ( siehe auch Abb. 3). Die meisten Laserimpulse werden von der Geländeoberfläche, von Gebäuden etc. vollständig reflektiert (single echo). Besonders durch die Vegetation werden Impulse geteilt und mehrfach reflektiert. Dabei werden i. d. R. die erste (first echo) und die letzte (last echo) Reflexion aufgezeichnet. Das DOM wird dabei aus allen first echoes aufgebaut. Das DGM hingegen wird aus allen Bodenpunkten berechnet und setzt daher eine Klassifiziierung der Punktwolke in Boden- und Nicht-Bodenpunkte voraus. Dieser auch als Filterung bezeichnete Prozess wird größtenteils automatisch berechnet, eine fachkundige Beurteilung der Ergebnisse und eine manuelle Korrektur sind aber unablässig. Die Qualität eines DGM für hydraulische Anwendungen ist also nicht nur durch die Genauigkeit der Punktwolke, sondern auch in besonderer Weise durch die Güte der Datenfilterung festgelegt.

\section{Prüfung der ALS - Daten}

Die Genauigkeit des Geländemodells ist wesentlich für die Qualität von 2D-Hochwasserabflussuntersuchungen. Die Ergebnisse aus der Befliegung sind sehr abstrakt (digitale Punktwolken) und die gelieferten Datenmengen sehr groß. Daher wurden im Auftrag der Steiermärkischen Landesregierung die Daten der ALS-Befliegungen durch ein externes Vermessungsbüro kontrolliert. Folgende Kriterien wurden geprüft:

1. Vollständigkeit der Teil- und Schlusslieferung 
2. Aufnahmedichte der ALS Punktwolke (besser als 1 Punkt je $\mathrm{m}^{2}$ )

3. Streifenkontrolle: Differenzen in den Überlappungsbereichen der Flugstreifen

4. Kontrolle der Transformation von WGS84 nach Gebrauchskoordinatensystem (Gauß-Krüger)

5. Vermessung von Kontrollflächen

6. Kontrolle der Lage- und Höhengenauigkeit durch Vergleich der Kontrollflächen mit dem gelieferten DGM

7. Visuelle Überprüfung der Datenfilterung durch Vergleich vor Ort

Die häufigsten Mängel waren:

- Transformation in das Gebrauchskoordinatensystem: Die Nichtberücksichtigung der Inhomogenitäten des österreichischen Festpunktfeldes und der Geoidundulationen im alpinen Bereich führte zu Höhenfehlern.

- Höhengenauigkeit: Die geforderte Höhengenauigkeit von $+/-15 \mathrm{~cm}$ wurde in einigen Bereichen nicht erreicht, während die Punktdichte und die Lagegenauigkeit eingehalten wurde.

- Vollständigkeit der Lieferung: Die Vollständigkeit des geforderten Lieferumfanges war nicht immer gegeben.

Abbildung 3 zeigt ein Differenzenmodell, das zur Kontrolle der Höhengenauigkeit herangezogen wurde (links) sowie Kontrollmessungen (rechts).

\section{Anforderungen an die \\ ALS Daten hinsichtlich der Abflussmodellierung}

ALS Daten stellen eine moderne und sehr genaue Möglichkeit zur Erfassung und Modellierung von Vorlandbereichen in Hochwasserabflussgebieten dar. Im Vergleich zu Abflussmodellierungen ohne ALS, bei denen üblicherweise zwischen Profilaufnahmen interpoliert wird, stellen die ALS-Daten einen großen Genauigkeitsgewinn dar. Auf die Aktualität der Daten hinsichtlich zwischenzeitlich getätigter Geländeveränderungen bzw. Hochwasserschutzmaßnahmen ist dabei zu achten. Erfahrungen zeigen, dass unter anderem aufgrund der großen Genauigkeitsansprüche an die Ergebnisse der 2D-Abflussmodellierung auf die Aktualität, insbesondere bei Schüttungen im Abflussbereich besonders zu achten ist. Eine Herausforderung stellt dabei die laufend erforderliche Aktualisierung der Hochwasserabflussbereiche dar.

Im Zuge der Qualitätssicherung des Digitalen Höhenmodells (DHM), welches

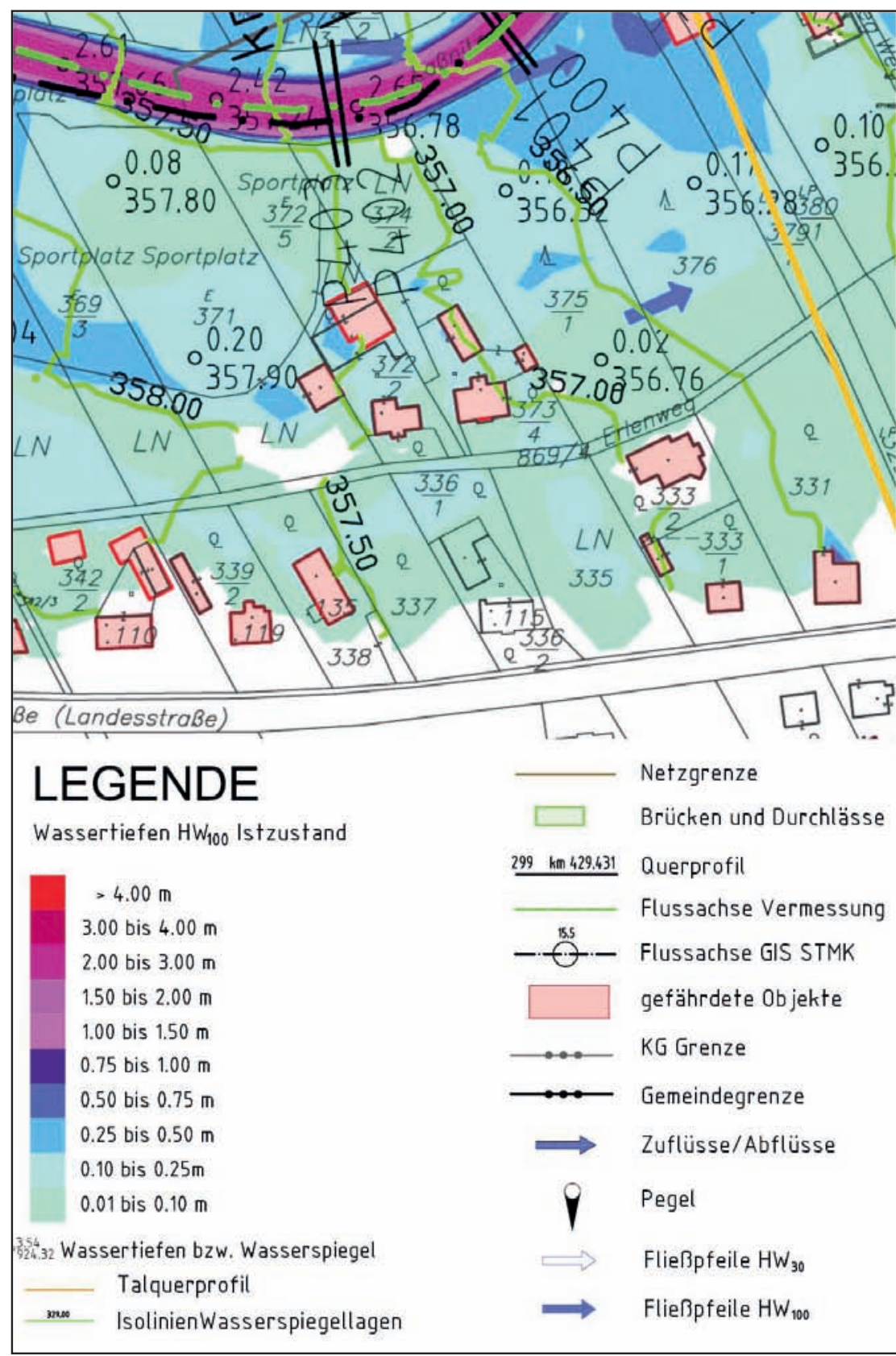

Abb. 2: Beispiel für die Darstellung der Wassertiefen bei HQ100.

aus ALS Daten und terrestrischen Vermessungsdaten erstellt wird, stellen sich folgende Fragen:

1. Mit welchen Arbeitschritten und Arbeitsmethoden wird in der Vorbereitung der terrestrischen Vermessung sichergestellt, dass - bei Einhaltung der geforderten Genauigkeiten - das aus ALS und terrestrischer Vermessung resultierende DHM vollständig und homogen ist? Auf folgende Punkte ist diesbezüglich u. a. zu achten:

- Kontrolle der ALS Daten, ob Flächen mit dichter Vegetation und
Bruchkanten - v. a. Uferborde - ausreichend erfasst wurden

- Sicherstellung der Homogentität des DHM z.B. durch Verwendung von gemeinsamen Datengrundlage (Festpunkte, Kontrollflächen, etc.) bei der terrestrischen Vermessung

- Festlegung der terrestrisch zu vermessenden Bruchkanten, Profile Objekte, etc. gemeinsam mit dem Hydrauliker

- Plausibilitätsprüfung und Kontrolle im Gelände 


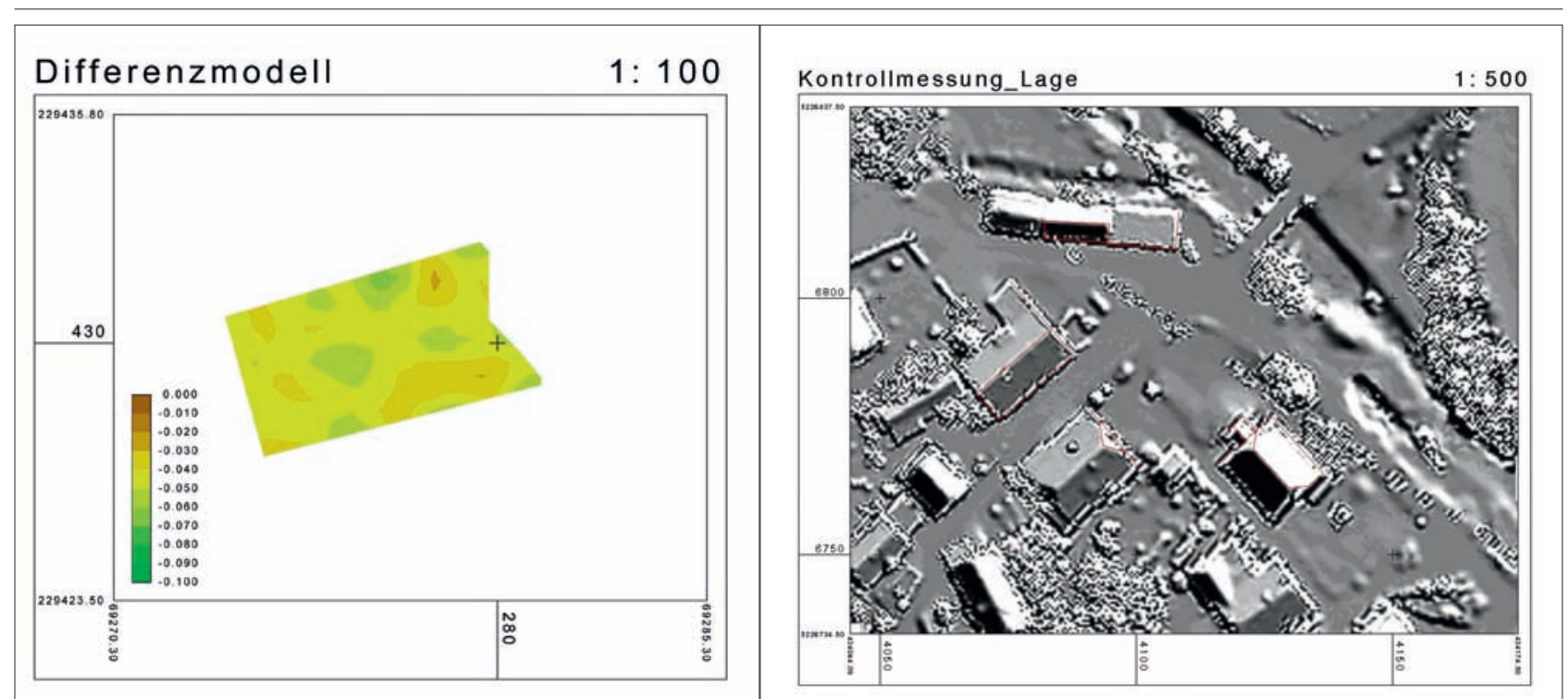

Abb. 3: Ergebnisse der Höhen- und Lagekontrolle. Links: Differenzenmodell (Höhen in m); Rechts: DGM mit Kontrollmessungen.

2. Mit welchen Arbeitsschritten und Arbeitsmethoden wird im Fluss und im Vorland die Qualität des DHM, welches für die hydraulische Berechnung verwendet wird, sichergestellt? Auf folgende Punkte ist diesbezüglich u. a. zu achten:

- Eventuell Mitverwendung der ALS Daten für die Modellierung im Flussschlauch und im Böschungsbereich

- Kontrollierte Ausdünnung der ALS Daten im Vorland (eventuell gemeinsam mit dem Hydrauliker)

- Kontrolle der Ausdünnung über einen Differenzenplan

Die 2D-Abflussuntersuchungen umfassen folgende Arbeitspakete ( $A b b .4)$ :

- Übernahme Laserscan - terrestrische Vermessung - Digitales Höhenmodell

- Erstellung eines homogenen digitalen Höhenmodells als Grundlage für die hydraulische Berechnung und schutzwasserwirtschaftliche Maßnahmen

- Hydrologische Grundlagen

- Verifizierung von Pegelschlüsselkurven

- Hydrologischer Längenschnitt

- Abschätzung von Hochwasserwellen

- Berechnung der Hochwasseranschlaglinien

- Stationäre Berechnung HQ30/ $100 / 300$

- Instationäre Berechnung HQ30/ 100

- Darstellung der Überflutungsflächen, Wassertiefen, Strömungsge- schwindigkeiten und -vektoren

- Konsensüberprüfung

- Gegenüberstellung der aktuellen Abflusskapazität mit dem Rechtsbestand

- Risikoabschätzung

- Charakterisierung des Überflutungsrisikos der gefährdeten $\mathrm{Ob}$ jekte

- Technisches Maßnahmenkonzept

- Genereller Maßnahmenvorschlag mit Darstellung der Auswirkung

- Projektsmanagement und Öffentlichkeitsarbeit

- Interne und externe Qualitätskontrolle

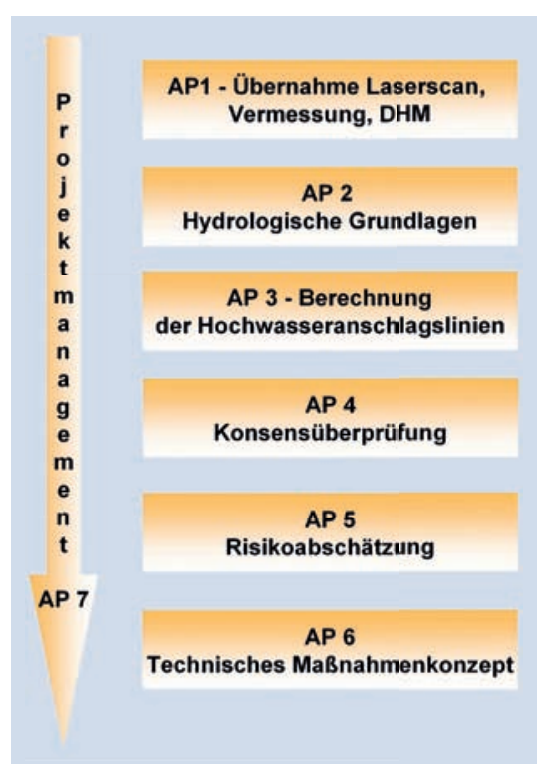

Abb. 4: Ablaufschema der Abflussuntersuchungen

\section{Anwendung der ALS-Daten für die Erstellung des Digitalen Höhenmodells (DHM)}

ALS-Daten werden sowohl bei der Modellierung des Flussschlauches als auch des Vorlandes für die hydraulischen Berechnungen verwendet. Die Genauigkeit des DHM ist von der tatsächlichen Punktdichte am Boden und von der Topographie des Geländes abhängig.

\section{Flussschlauch}

Die Modellierung des Flussschlauches erfolgt primär unter Verwendung der terrestrisch vermessenen Querprofile. Besonders wichtig für die hydraulische Berechnung des Abflusses sind die Uferborde. Sind diese in der Natur eindeutig definiert (Dämme, Mauern etc.), ist die terrestrische Vermessung vorzuziehen. Im natürlichen Gelände aber ist es effizienter, die Uferborde unter Verwendung der ALSDaten festzulegen. In den Schummerungen und Höhenschichtenbildern erkennt man Senken wesentlich besser als vor Ort bei meist dichtem Bewuchs (Abb. 5).

Die wesentlichen Stützpunkte für die Festlegung der Uferborde aus den ALS-Daten sind die terrestrisch vermessenen Punkte in den Querprofilen. Durch den Vergleich der terrestrischen Punkte im Vorland mit den ALS-Daten hat man eine sehr gute Aussage über die Höhengenauigkeit der ALS-Daten in diesem Bereich. Die Erfahrung aus den letzten Projekten hat gezeigt, dass bei sorgfältiger Prozessierung der ALS-Daten die Standardabweichung der Höhenfehler bei ca. $+/-5 \mathrm{~cm}$ liegt. 


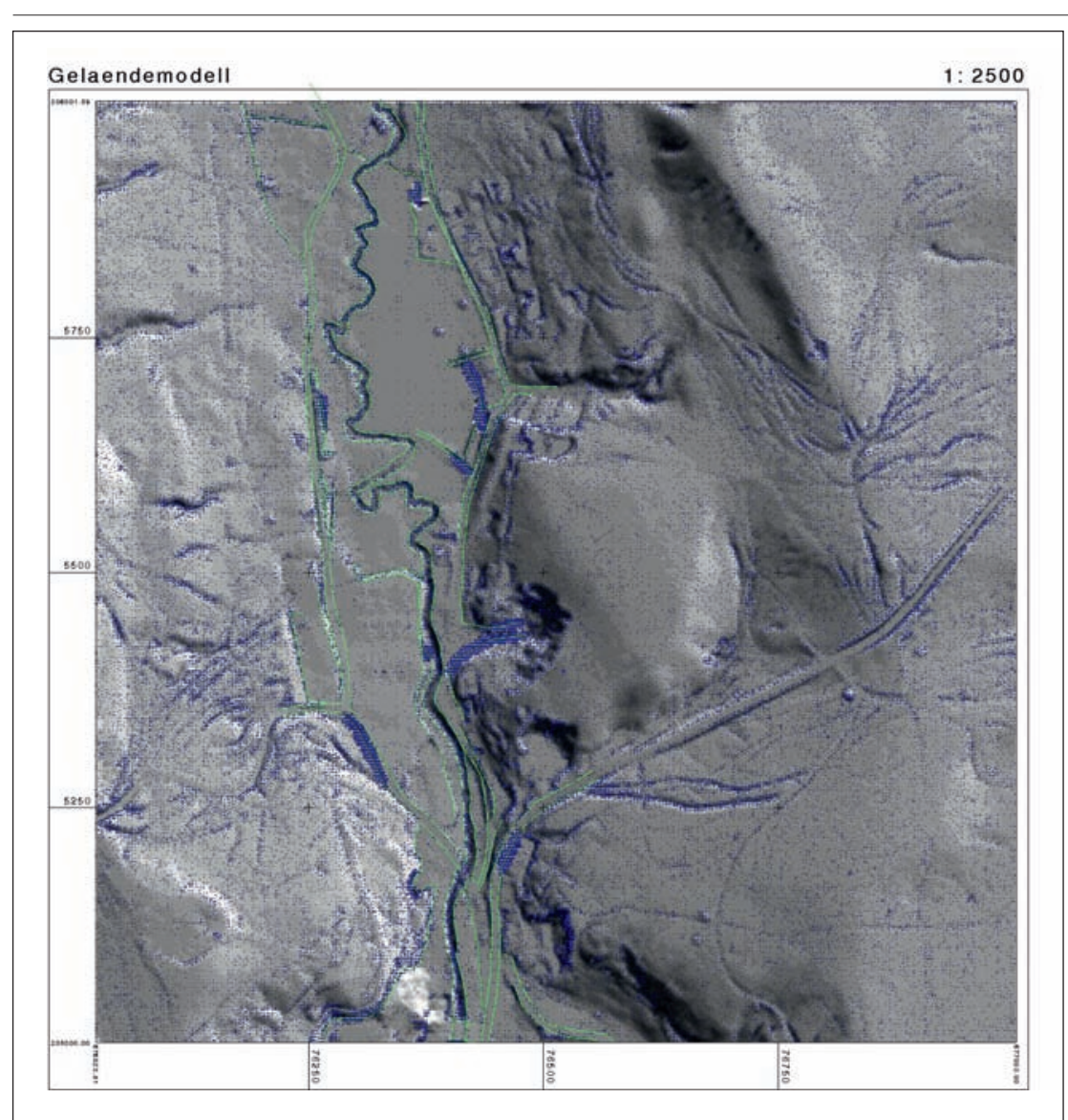

Abb. 5: Schummerung eines DGM mit Bruchkanten und ausgedünnter Punktwolke.

Eine weitere wesentliche Grundlage für die Modellierung des Flussschlauches ist die Gewässerachse, die ebenfalls mittels terrestrischer Profilpunkte und ALS-Daten definiert wird. Die Lagegenauigkeit der Gewässerachse aus den ALS-Daten ist dabei wesentlich besser als die Festlegung der Achse mittels zweidimensionaler digitaler Orthophotos.
Vorland gung - 1 bis 4 Punkte je $\mathrm{m}^{2}$ - und im Digitalen Geländemodell (DGM) - Rasterweite $1 \mathrm{~m} \times 1 \mathrm{~m}$ - ist für die Berechnungsprogramme zu groß. Außerdem sind für die Beschreibung des Geländemodells die Bruchkanten besonders wichtig. Daraus leiten sich zwei wesentliche Arbeitsschritte ab:
Die Anzahl der Punkte aus der ALS Beflie-
- Ableitung der Bruchkanten aus den Daten der ALS-Befliegung oder aus dem DGM, wobei die Berechnung unter Verwendung der originalen ALSMessdaten bessere Ergebnisse liefert als die Berechnung mittels DGM.

Qualifizierte Ausdünnung des DGM. Dazu müssen zuerst die Bruchkanten in das DGM integriert werden um danach ein neues DGM mit wesentlich weniger Punkten und daher größeren Punktabständen zu interpolieren. Die Kontrolle dieses Prozesses erfolgt durch die Berechnung eines Differenzenmodells, in dem die Höhendifferenzen zwischen originalem und ausgedünntem Modell farblich dargestellt sind.

\section{Korrespondenz:}

Dipl.-Ing. Peter Rauchlatner

Amt der Steiermärkischen Landesregierun

FA19A - Wasserwirtschaftliche Planung und Siedlungswasserwirtschaft

Stempfergasse $5-7$

$8010 \mathrm{Graz}$

Tel.: +43 316/ 877-2022

Fax: +43 316/ 877-2480

E-Mail: peter.rauchlatner@stmk.gv.at

Dipl.-Ing. Wolfgang Höppl Vermessungsbüro Höppl Mannagettaweg 18

A-8010 Graz

Tel.: +43 316/328252

Fax: +43 316/328252 25

E-Mail: w.hoeppl@geo-hoeppl.at

\section{LITERATUR}

Mandlburger G, Höfle B, Briese Ch, Ressl C, Otepka J, Hollaus M, Pfeifer N (2009) Topographische Daten aus Laserscanning als Grundlage phische Daten aus Laserscanning als Grundlage
für Hydrologie und Wasserwirtschaft. Österr. für Hydrologie und Wasserwirtschaft. Oster. 